\title{
Dysfunctional Profile for Patients in Physical Neurorehabilitation of Upper Limb
}

\author{
M.A. Villán-Villán $\quad$ R. Pérez-Rodríguez C. Gómez , E. Opisso J.M. Tormos- \\ J. Medina and E.J. Gómez Aguilera
}

\begin{abstract}
This paper proposes a first approach to Objective Motor Assessment (OMA) methodology. Also, it introduces the Dysfunctional profile (DP) concept. DP consists of a data matrix characterizing the Upper Limb (UL) physical alterations of a patient with Acquired Brain Injury (ABI) during the rehabilitation process. This research is based on the comparison methology of UL movement between subjects with ABI and healthy subjects as part of OMA. The purpose of this comparison is to classify subjects according to their motor control and subsequently issue a functional assessment of the movement. For this purpose Artificial Neural Networks (ANN) have been used to classify patients. Different network structures are tested. The obtained classification accuracy was $\mathbf{9 5 . 6 5 \%}$. This result allows the use of ANNs as a viable option for dysfunctional assessment. This work can be considered a pilot study for further research to corroborate these results.
\end{abstract}

Keywords-Dysfunctional Profile, Classification, Physical Neurorehabilitation, Objective Assessment, Upper Limb.

\section{INTRODUCTION}

Acquired brain injury ( $\mathrm{ABI})$ is a brain structure lesion produced suddenly after birth. The cause of ABI can be either traumatic (road-traffic accidents, falls, etc.) or nontraumatic (strokes, brain tumors, infections, etc.). The most common ABIs are cerebrovascular disease (stroke) and traumatic brain injury (TBI) [1], [2]. These injuries, due to their physical, sensory, cognitive, emotional and socioeconomic consequences, considerably change the life of both patients and families [3].

According to predictions by the World Health Organization, $\mathrm{ABI}$ will be among the ten most common causes of disability by the year 2020. This injury is the second leading cause of death and the eighth cause of severe disability in the elderly. Every year, nine million people suffer from stroke in the world [4] while 100.000 new cases in Spain, mostly people over 65 years old [5]. In USA, it is estimated that 5.3 million Americans, more than $2 \%$ of the country, are currently disabled due to TBI [6]. The annual incidence to TBI in Europe is estimated to be 235 per 100000 population [7].
The ABI patients with functional alterations need treatments to reduce disability and improve their quality of life. Neurorehabilitation is a clinical process whose main objectives are restoring, minimizing or compensating the impairments in people with disabilities of neurological origin. The physical neurorehabilitation is intended to provide the patients with the capacity to perform specific activities of daily life (ADL) required for an independent life in terms of the handling of objects in the environments in which the upper limb (UL) is directly involved [8].

Assessment methods of UL motion depend on clinician experience and subjectivity. The UL functional evaluations are focused on clinical tests highly dependent on the examiner's criteria and require a clinician to score the performance of patients in some tasks on specific scales. Some of the most used clinical tests are: Fugl Meyer (FM) [9], Action Research Arm Test (ARAT) [10] and Chedoke-Mc Master Stroke Assessment [11].

The dysfunctional motion modeling of patients undergoing a rehabilitation treatment has been addressed by different methods based on kinematic analysis of UL movement [12-14]. Kinematic analysis provides objective data that allows making accurate descriptions of UL movements and activities executed by healthy subjects for establishing normal pattern which would be used in the comparison with pathological subjects [8]. Some methods focused on the comparison of movement between patients and healthy subjects [12]. Other methods developed and validated a $3 \mathrm{D}$ upper limb model on a motion assessment system that compares kinematics of the joint between the affected and unaffected arms $[13,14]$.

Kinematic models of healthy subjects UL motion in the execution of ADL have been developed in scientific literature [15]. Due to lack dysfunctional Kinematic models, it is required to create new methods that allow making an accurate, precise and reliable assessment of UL motion, in order to provide objective data for the interpretation of the motor function of the patients. To solve the lack of dysfunctional kinematic models, the Dysfunctional Profile (DP) concept is proposed. DP consists of a data matrix characterizing the 
physical alterations of each patient. This profile is dynamic and can be updated during the rehabilitation process, allowing the tracking of the patients' evolution in a controlled fashion.

Previous authors' research is focused on the design of new methodologies to generate a change in the current model of UL physical neurorehabilitation, to improve the efficacy of treatment, personalize it and base it on evidence $[3,8,16]$. DP can be used in applications such as: 1 . to allow robotic anticipatory assistance [16], and 2. to design an Objective Motor Assessment (OMA) methodology. The OMA methodology is conceived to provide an objective measurement of patient's DP. This assessment would assign an individual score for each patient that indicates the level of motor function the degree of similarity between the UL motion of the patient and the healthy subjects. OMA methodology consists of three consequential stages: 1 . Calculation, 2.Classification and 3.Evaluation.

This paper is focuses on the stages 1 and 2 of the OMA methodology. The main goal of this research is to make a first approach to OMA based on classification of subjects according to their motor control in the execution of ADLs using the Artificial Neural Network (ANN).

\section{MATERIALS AND METHODS}

Fig.1. shows the proposed methodology diagram. First, kinematic analysis of UL movement is performed. Second, Principal Component Analysis (PCA) is applied in order to reduce the data dimension. Then, a Multilayer Perceptron (MLP) has been used for classifying subjects according to their dysfunctional deficits. The architecture of the network is established empirically.

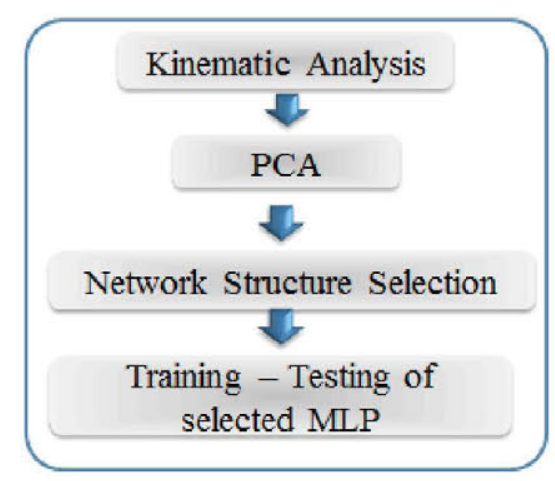

Fig.1 Proposed Methodology

\section{A. Material}

The BTS-SMART-D system [17] has been used to obtain all motion data of healthy subjects and ABI subjects. This device is a digital optoelectronic system with 6 infrared cameras with frequency of $140 \mathrm{~Hz}$ and resolution of $1.4 \mathrm{Mp}$. Motion capture was performed with a model bimanual sixteen points [18]. The mathematical calculations of metrics and network training have been performed with the tool MATLAB ${ }^{\circledR} \mathrm{r} 2009 \mathrm{~b}$ running on a computer with a $2.4 \mathrm{GHz}$ Intel ${ }^{\circledR}$ Core ${ }^{\mathrm{TM}}$ Duo processor with a 4 GB RAM has been used. The PCA is performed with Statistic PASW v 18 (SPSS).

\section{B. Biomechanical Model}

A kinematic chain with three segments is used in this proposal. This biomechanical model consist of six degrees of freedom (DoF): three in the shoulder joint (flexion/extension, abduction/adduction and rotation), two in the elbow joint (flexion/extension and pronation/ supination) and one in the wrist joint (flexion/extension) [8].

\section{Calculation of Kinematic Variables}

To make a more accurate kinematic analysis of the patients UL motion, an ADL modeling methodology [19] has been applied. Therefore, for each ADL that the patient executes, a state-chart diagram is obtained. Following this methodology, the activity gets partitioned into several transitions that are studied independently.

Fig. 2 shows the state diagram of the "picking up a bottle" ADL with three transitions. T1: Rest - bottle pick up, T2: bottle pick up - bottle on the table and T3: bottle on the table - rest.

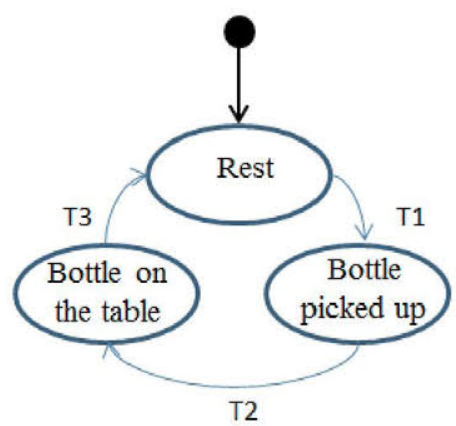

Fig. 2 State diagram of the "picking up a bottle" ADL

The temporal and spatial parameters, distributed in four metrics, were calculated in each transition of the ADL so that in each metric at least 3 Motion Parameters (MP), can be obtained. Below are the metrics and the MPs obtained in each transition: 
$\checkmark \quad$ Time (s): time used to execute the ADL. 3 MPs.

$\checkmark$ Tangential velocity peak $(\mathrm{mm} / \mathrm{s})$ : maximum value of the rate of change of the position of the End Effector (EE) of human UL per second. 3 MPs.

$\checkmark$ Angular velocity peak (deg/s): maximum value of the rate of change of angle in each joint DoF per second. 18 MPs.

$\checkmark$ Trajectory length index (TLI): the ratio of the length of the actual path travelled by the EE in space $(\mathrm{T})$ to the length of the straight line joining the initial and final end-point positions (D) [12]. 3 MPs.

$$
T L I=\frac{T}{D}
$$

\section{Classification of Subjects}

MLP is the most commonly used feedforward ANN [20]. In this study, MLPs used consisted of: $n$ neurons in the Input layer (I) (one for each MP), a single neuron in Hidden layer $(\mathrm{H})$, a neuron in Output layer $(\mathrm{O})$ (category to which an individual belongs). Backpropagation learning [1], was used with a hyperbolic tangent as an activation function for neurons $\mathrm{H}$ and a lineal function for neurons in $\mathrm{O}$. Therefore, when motor function of a subject is assessed, the algorithm can distinguish the category the subject belongs to. The categories according to the control of motor function are: Healthy subject's Category (HC) and Patients with ABI Category (PC).

\section{EXPERIMENTAL WORK}

For training and testing of the different MLPs, data from the "picking up a bottle" ADL have been used. Fig. 3 shows frontal view of this ADL: an empty plastic bottle with a capacity of $330 \mathrm{ml}$ is located on a shelf that is placed on a table. The subject is asked to put the bottle in the closest right corner of the table. The exact place is indicated by a solid dot. This ADL has been designed by therapists of the Guttmann Institute.

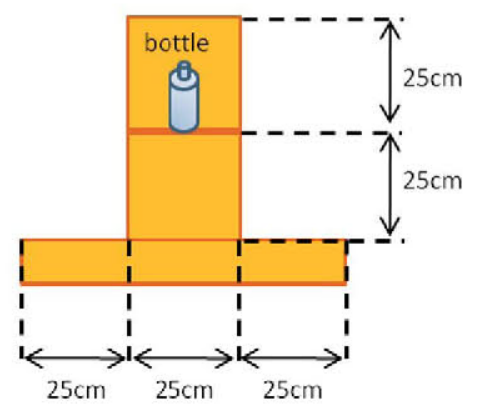

Fig. 3 Frontal view of the "picking up a bottle" ADL setup
The dataset used in this research consisted of 45 subjects divided into: $40 \mathrm{HCs}$ and 5 PCs. To assess the proposed method, the network was trained with $48 \%$ of the data, of which 20 belonged to HCs and 2 belong to PC. The network was tested with $20 \mathrm{HC}$ and $3 \mathrm{PC}$ representing the $52 \%$ of data. Different structures and parameters were tested to determine the best network structure.

\section{RESULTS AND DISCUSSION}

In the proposed method PCA of the input data was carried out (27 MP previously obtained) minimizing the loss of information. The number of initial MP was reduced to 10 . These MP contain $90 \%$ of the original data information.

Table 1 shows the three best results with different network structures. Mean Squared Error (MSE) and Learning Rate (LR) are the parameters of network training, and accuracy in Classification $(\% \mathrm{C})$ is the result. The best result in classification accuracy was $95.65 \%$. Results 2 and 3 obtained the same classification accuracy with similar architectures but different network parameters (MSE and LR). The difference between them and the reason for selecting the network structure 3 as the best result is because with this structure 10-12-1 (I-H-O) and network parameters 0.001 (MSE) and 0.7 (LR), the MLP classified $100 \%$ of HC. Additionally, with a small MSE the possibility that the network makes a mistake in classification is minimized because it approaches zero.

Table 1 The three best obtained results with different network structures and parameters.

\begin{tabular}{ccccccc}
\hline \hline Results & $\mathrm{I}$ & $\mathrm{H}$ & $\mathrm{O}$ & MSE & LR & \% C \\
\hline 1 & 10 & 6 & 1 & 0.1 & 0.01 & 86.95 \\
\hline 2 & 10 & 12 & 1 & 0.1 & 0.05 & 95.65 \\
\hline $\mathbf{3}$ & $\mathbf{1 0}$ & $\mathbf{1 2}$ & $\mathbf{1}$ & $\mathbf{0 . 0 0 1}$ & $\mathbf{0 . 7}$ & $\mathbf{9 5 . 6 5}$ \\
\hline \hline
\end{tabular}

The results indicate that the use of a MLP can be useful tool for subjects classifying as part of OMA in physical neurorehabilitation of UL. Also, obtained results are in line with research claiming that the classification accuracy improves with ANNs when the PCA is used as method of feature extraction and selection [21].

Table 2 shows the confusion matrix of the best classification achieved by the selected MLP (result 3). The network classified $100 \% \mathrm{HC}$ and $66.67 \% \mathrm{PC}$.

Table 2 Confusion matrix of the best classification achieved by the proposed method.

\begin{tabular}{ccccc}
\hline \hline & HC & PC & Samples & Error \\
\hline HC & 20 & 0 & 20 & 0 \\
\hline PC & 1 & 2 & 3 & 1 \\
\hline \hline
\end{tabular}


Although the results are promising, they are inconclusive, because one of the limitations of this research is the amount of movement data available for subjects with ABI, due to technical and clinical limitations necessarily present in this type of experimental studies. It is noted that with a greater amount of patient data, these results may vary, possibly increasing the classification accuracy. Therefore, this research can be considered as a first step in the development of the OMA methodology.

\section{CONCLUSIONS}

This research proposed a model based on a MLP for the classification of the level of dysfunction of ABI subjects and healthy subjects. The PCA was applied for the data dimensionality reduction (allowing for a choice of an appropriate data set for network training), to reduce the number of MP, increase classification accuracy, the lowest computational cost and the network training time reduction. The MLP enables assessment of motor function of subjects and the sorting of the category to which they belong ( $\mathrm{HC}$ and PC). The results obtained by the MLP with the proposed method are promising. All healthy subjects are well classified and subjects with ABI are well classified in 2 of the 3 patients. This research can be considered as a first step in the development of a method that allows the objective assessment of UL motor control of patients undergoing a physical rehabilitation treatment.

Due to the fact that patients' motor function evolve over time, allowing the tracking of objective data during the rehabilitation treatment, DP of each patient could be continuously updated. Thereby, a useful database for medical research would be generated. Therefore, the implementation of OMA could optimize physical rehabilitation process. On the other hand, adding information to the database, such as patients' demographic data and tests results, it could be possible to create data exploitation and analysis system that focuses on extracting knowledge to improve the effectiveness of rehabilitation treatments, in a personalized way and based on evidence. In addition, new tools and applications for medical help and support platforms such as a management platform and therapeutic planning could be designed in the future.

\section{REFERENCES}

1. Güler I, Gökçil Z, \& Gülbandilar E (2009) Evaluating of traumatic brain injuries using artificial neural network. Expert Syst. Appl. 36(7):10424-10427 DOI 10.1016/j.eswa.2009.01.036

2. Murray CJL, Lopez AD (1997) Alternative projections of mortality and disability by cause 1990-2020: Global Burden of Disease Study. Lancet, 349(9064):1498-1504 DOI 10.1016/S0140-6736(96)07492-2

3. Pérez R, Marcano A, Costa U, et al. (2012) Inverse kinematics of a 6 DoF human upper limb using ANFIS and ANN for anticipatory actuation in ADL-based physical Neurorehabilitation. Expert Syst. Appl. 39(10):9612-9622 DOI 10.1016/j.eswa.2012.02.143
4. World Health Organization, Burden of Disease Statistics, at http://www. who.org

5. Alvaro LC, Lopez-Arbolea P, Cozar R (2009) Hospitalizations for acute cerebrovascular accidents and transient ischemic attacks in Spain: temporal stability and spatial heterogeneity, 1998-2003. Revi. Calid. Asist. 24(1):16-23 DOI 10.1016/S1134-282X(09)70071-5

6. Brain Injury Association of America, at http://www.biausa.org

7. The Lancet Neurology (2010) Traumatic brain injury: time to end the silence. The Lancet Neurology 9(4):331 DOI 10.1016/S14744422(10)70069-7W

8. Pérez R, Costa Ú, Torrent M, et al. (2010) Upper Limb Portable Motion Analysis System Based on Inertial Technology for Neurorehabilitation Purposes. Sensors 10:10733-10751 DOI $10.3390 / \mathrm{s} 101210733$

9. FuglMeyer A, Jaasko L, Leyman I, et al. (1975). The post stroke hemiplegic patient. A method for evaluation of physical performance. Scand J Rehabil Med 7(1):13-31

10. McDonnell M (2008) Action Research Arm Test. Aust. J Physiother. 54(3):220-221.

11. Gowland C, Stratford P, Ward M, et al. (1993) Measuring physical impairment and disability with the Chedoke-McMaster Stroke Assessment. Stroke 24(1):58-63 DOI 10.1161/01.STR.24.1.58

12. Cirstea MC, Levin MF (2000) Compensatory strategies for reaching in stroke. Brain 123(5):940-953 DOI 10.1093/brain/123.5.940

13. Hingtgen BA, MeGuire JR, Wang M, Harris GF (2006) An upper extremity kinematic model for evaluation of hemiparetic stroke. J Biomech 39(4):681-688 DOI 10.1016/j.jbiomech.2005.01.008

14. Hingtgen BA, McGuire, JR, Wang M, et al. (2004) Quantification of reaching during stroke rehabilitation using unique upper extremity kinematic model. In Proceedings of the 26th Annual International Conference of the IEEE EMBC, San Francisco, USA, 2004, vol. 7, pp 4916-4919

15. Costa U, Opisso E, Perez R, et al. (2010) 3D motion analisys of activities of daily living: implication in neurorehabilitation. In Proceedings of the International Gait and Clinical Movement Analysis Conference, Miami, USA, 2010

16. Peréz R, Costa Ú, Rodríguez C, et al. (2013) Assistance-as-needed robotic control algorithm for physical neurorehabilitation. In Proceedings of the International Conference on Recent Advances in Neurorrhabilitation, Valencia, Spain, 2013, pp 138-142

17. BTS Bioengineering, at http://www.btsbioengineering.com

18. Rab G, Petuskey K, Bagley A. (2002) A method for determination of upper extremity kinematics. Gait\&Posture 15(2):113-119 DOI 10.1016/S0966-6362(01)00155-2

19. Pérez R, Costa Ú, Solana J, et al. (2009) Modelado de Actividades de la Vida Diaria para Neurorrehabilitación Funcional de miembro superior. In CASEIB 2009, In Proceedings of the XXVII Annual Congress of the Spanish Biomedical Engineering Society, Cádiz, Spain, 2009

20. Haykin S. (1994) Neural networks, a comprehensive foundation book. Macmillan College Publishing Company, New York.

21. Burcu E, Tulay Y (2008) Improving classification performance of sonar targets by applying general regression neural network with PCA. Expert Syst. Appl. 5:472-475 DOI 10.1016/j.eswa.2007.07.021
Author: Mailin Adriana Villán Villán (M.A. Villán-Villán)
Institute: ETSI Telecomunicación, Universidad Politéenica de Madrid
Street: Avenida Complutense 30
City: Madrid
Country: Spain
Email: mvillan@gbt.tfo.upm.es 